\title{
Groupwise Deformable Registration of Fiber Track Sets using Track Orientation Distributions
}

\author{
Daan Christiaens, Thijs Dhollander, Frederik Maes, Stefan Sunaert, and \\ Paul Suetens
}

\begin{abstract}
Diffusion-weighted imaging (DWI) and tractography allow to study the macroscopic structure of white matter in vivo. We present a novel method for deformable registration of unsegmented full-brain fiber track sets extracted from DWI data. Our method attempts to align the track orientation distributions (TOD) of multiple subjects, rather than individual tracks. As such, it can handle complex track configurations and allows for multi-resolution registration. We validated the registration method on synthetically deformed DWI data and on data of 15 healthy subjects, and achieved sub-voxel accuracy in dense white matter structures. This work is, to the best of our knowledge, the first demonstration of direct registration of probabilistic tractography data.
\end{abstract}

\section{Introduction}

Because of their unique ability to study the macroscopic structure of white matter (WM) in vivo, diffusion-weighted imaging (DWI) and tractography have become important tools in neuroscientific research. Probabilistic tractography methods on high angular resolution diffusion imaging (HARDI) data have been shown to be robust to noise and complex fiber configurations such as crossings, but the large amount of tracks they typically generate are difficult to analyse manually. Automated analysis, such as clustering and labelling of tracks into anatomical bundles $[9,13,20]$

D. Christiaens · T. Dhollander · F. Maes · S. Sunaert · P. Suetens

Medical Imaging Research Center (MIRC), KU Leuven,

Herestraat 49 box 7003, 3000 Leuven, Belgium

e-mail: daan.christiaens@esat.kuleuven.be

D. Christiaens · T. Dhollander · F. Maes $\cdot$ P. Suetens

iMinds-MIC, Department of Electrical Engineering (ESAT), KU Leuven, Belgium

S. Sunaert

Translational MRI, Department of Imaging \& Pathology, KU Leuven, Belgium 
and population-based variability analysis [8], is facilitated by the availability of a population atlas of the expected track configuration. While such atlases can be built by registration of anatomical images of different subjects, it has been pointed out $[8,12]$ that direct registration of the extracted tractograms may be better suited for population-based analysis of the track configuration, as the matching criterion is directly related to the structures of interest.

Current methods for non-rigid track-based registration (e.g., $[2,8,21]$ ) require a pre-existing segmentation of WM bundles. Registration of unsegmented, full-brain tractography data has, to our knowledge, been limited to affine transformations [11, $12,22]$. Moreover, the effectiveness of these methods has only been demonstrated on deterministic, DTI-based tractography. Secondly, many of these methods are based on distance measures between a compact set of sample tracks [11,12], feature points [2] or "fiber modes" [22], and thereby neglect part of the information contained in dense track sets. A notable exception is the metric on currents [8], in which the distance is defined based on the spatial distribution of the mean local track direction. In regions of crossings or track dispersion, however, this mean track direction is ill-defined.

In this paper, we examine the use of an image-domain representation of the spatial and angular distribution of full-brain track sets for deformable registration and atlas construction. In contrast to the current state-of-the-art, our method does not depend on a pre-existing bundle segmentation and is able to handle crossings and dispersion. We evaluate our method on artificially deformed data of a single subject (known ground-truth) and on HARDI-based probabilistic tractography data of 15 normal subjects, and compare the results to image registration methods based on the fractional anisotropy (FA) and the fiber orientation distribution (FOD).

\section{Methods}

\subsection{Track Orientation Distribution}

The core idea of this paper is to represent the tractogram as a probability distribution function (PDF) in the image domain, rather than a set of individual tracks (samples from this distribution). One example of such representation is the track-density image (TDI) [3], in which the intensity of each voxel is determined by the number of tracks that cross that voxel. In recent work, our group has generalized the TDI to incorporate angular information as well [5]. As such, we provide a full spatio-angular PDF of the tractogram, named the track orientation distribution (TOD).

Suppose each track $t$ that crosses a voxel $\vec{r}$ is linearly parametrized by $\varepsilon$ according the length along the track, and $\varepsilon_{1}$ and $\varepsilon_{2}$ are the values of this parametrisation at the voxel boundaries. The TOD in that voxel is a function in $\vec{u} \in \mathbb{S}^{2}$ and is formally defined as

$$
\operatorname{TOD}(\vec{r}, \vec{u})=\sum_{t} \int_{\varepsilon_{1}}^{\varepsilon_{2}} \frac{\tilde{\delta}_{\vec{z}_{t}(\varepsilon)}(\vec{u})}{\varepsilon_{2}-\varepsilon_{1}} \mathrm{~d} \varepsilon
$$




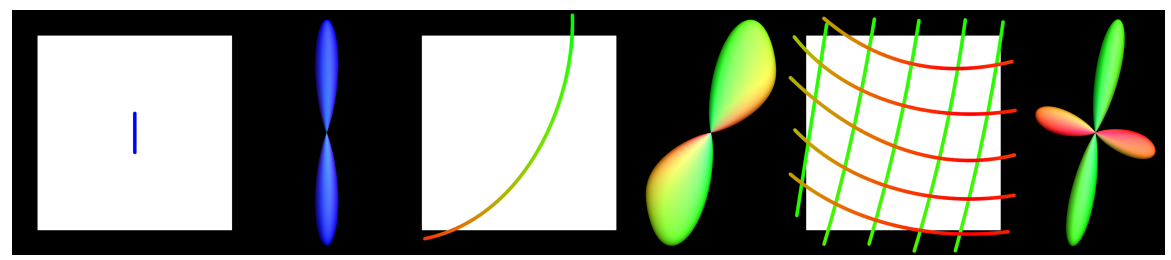

Fig. 1 Illustration of the track orientation distribution of SH order 16 for 3 different track configurations: a single line segment (left), a curved track (middle) and a crossing of 2 fiber track bundles (right).

where $\vec{z}_{t}(\varepsilon)$ is the local direction of $t$ at position $\varepsilon$ and $\tilde{\delta}_{\vec{z}_{t}(\varepsilon)}(\vec{u})$ is the apodized point spread function (PSF) oriented along this direction. The apodized PSF [14] is the closest strictly-positive approximation of a Dirac $\delta$-function in the spherical harmonics (SH) basis [4] of order $\ell_{\max }$, as shown in blue in Fig. 1. Hence, each track additively contributes to the TOD in a voxel by averaging the apodized PSF along its intersecting part (through integration and normalization).

Note that the TOD in Fig. 1 resembles the fiber orientation distribution (FOD) [17], but while they are of similar qualitative nature, they have an entirely different meaning [5]. The FOD estimates the local fiber direction from DWI data, whereas the TOD is a direct representation of any given tractogram, as generated by a given algorithm. Hence, the TOD incorporates more global information, while the FOD is directly related to the DWI data in a single voxel.

\subsection{TOD Registration and Reorientation}

The TODs of $N$ different subjects are represented as images of their coefficients in the $\mathrm{SH}$ basis of order $\ell_{\max }=4$. As such, the track set registration problem is redefined as a more convenient image registration problem. Inspired by existing work on FOD registration [15], we minimize the sum of squared differences (SSD) between all pairs of transformed TOD coefficients. By Parseval's theorem for spherical harmonics, this corresponds to minimizing the squared amplitude difference between the transformed TODs, integrated over $\mathbb{S}^{2}$.

In this study, we use the diffeomorphic demons algorithm [19], adapted for groupwise, multi-channel registration as in [6]. The SSD is minimized between the corresponding TOD coefficients $c_{i}[k]$ and $c_{i}[j]$ of all subject pairs $[k, j]$ by calculating the symmetric demons forces

$$
\vec{F}_{i}[k, j]=-\frac{\left(c_{i}[k]-c_{i}[j]\right)\left(\vec{\nabla} c_{i}[k]+\vec{\nabla} c_{i}[j]\right) / 2}{\left\|\left(\vec{\nabla} c_{i}[k]+\vec{\nabla} c_{i}[j]\right) / 2\right\|^{2}+\left(c_{i}[k]-c_{i}[j]\right)^{2} /(2 \eta)^{2}},
$$

where $\eta$ is the maximum step size. The total pairwise force $\vec{F}[k, j]$ is the (weighted) average of all channels, and the final force acting on subject $k$ is defined as the 
average force exerted by all other subjects, i.e.,

$$
\vec{F}[k]=\frac{1}{N-1} \sum_{\substack{j=1 \\ j \neq k}}^{N} \sum_{i} w_{i} \vec{F}_{i}[k, j] .
$$

The weights $w_{i}$ are defined such that the total weight of all coefficients of every order $\ell$ is the same, i.e., the 0 th order coefficient has weight $\frac{1}{3}$, the 5 coefficients of order 2 each have weight $\frac{1}{5 \cdot 3}$ and the 94 th order coefficients have weight $\frac{1}{9 \cdot 3}$. In our experience, these weights improve the robustness of the registration against challenging initialization. Note that as $\vec{F}[k, j]=-\vec{F}[j, k],(N-1)(N-2) / 2$ pairwise force fields need to be computed. The algorithm proceeds as in [19]: fluid regularization is applied by smoothing the total force field; the exponential of this update field is composed with the total deformation field; and finally diffusion regularization is applied by smoothing the total deformation field.

After each iteration, the TOD in every voxel is reoriented by applying the method of Raffelt et al. [14]. To this end, the TOD is decomposed into a mixture of apodized PSFs along equally distributed directions and reoriented by rotating and recomposing each of these PSFs, based on the Jacobian of the local deformation. In terms of computation time, reorienting the TOD is much more efficient than recomputing the TOD of the transformed tracks.

\section{Experiments and Results}

\section{Data, Processing and Fiber Tracking}

HARDI images of $N=15$ healthy subjects were acquired with a Siemens 3T system, using a twice-refocussed spin-echo sequence and an isotropic voxel size of $2.5 \mathrm{~mm}$. For each subject, DW images in 75 uniformly distributed gradient directions at $b=$ $2800 \mathrm{~s} / \mathrm{mm}^{2}$ and 10 non-DW images $\left(b=0 \mathrm{~s} / \mathrm{mm}^{2}\right)$ were acquired. Using MRtrix [18], we obtained the FODs of SH order 8 with constrained spherical deconvolution [17] and computed 2 million tracks for each subject using probabilistic streamline tracking with uniform seeding in a full brain mask, step size $0.2 \mathrm{~mm}$, minimum curvature radius $1 \mathrm{~mm}$, FOD amplitude cutoff 0.1 , and minimum track length $10 \mathrm{~mm}$.

\section{Experiment 1: Synthetically Deformed Single Subject}

In this first experiment, we aim to evaluate our registration method on tractography data of multiple deformed copies of a single subject with known anatomical correspondences. Therefore, we randomly selected 1 subject in the group, and warped and

reoriented its FODs onto each of the 14 other subjects according to the deformation 
fields obtained from groupwise demons registration of b0, FA and MD channels [6]. As such, we obtained 14 synthetic images and ground-truth deformation fields between each pair. We then generated fiber tracks in each of these images individually and computed the TODs. By deforming the FOD images, instead of the tractography data, this setup allows to test the algorithm's robustness against inter-subject differences in seeding and track density.

We ran the proposed registration method on the 14 synthetic TOD images using a multi-scale strategy. The registration starts with maximum step size $\eta$ and regularization kernel widths $\sigma_{\text {fluid }}$ and $\sigma_{\text {diff }}$ all equal to $5.0 \mathrm{~mm}$, then proceeds at $2.5 \mathrm{~mm}$ and finally at $1.25 \mathrm{~mm}$, with 500 iterations at each scale. In our experience, this is sufficient to ensure convergence. The resulting deformation fields and their inverse were composed in order to obtain all pairwise deformation fields. The accuracy of the registration is assessed by computing maps of the Euclidean distance between these deformations and their corresponding ground-truth. The mean of the distance maps of deformations that map onto the same subject then provides a measure of the registration accuracy of that subject.

In addition, we compared to the accuracy of direct image registration by performing the same groupwise demons algorithm with identical parameter settings on the FA (single channel) and on the FOD coefficients (cutoff at $\ell_{\max }=4$ ) of the 14 synthetic images. For comparability, all distance maps (defined in the space of the different images) are warped to the original atlas space, i.e., the atlas that was used to generate the synthetic data and the ground-truth deformations. As such, we can average all distance maps across subjects and properly compare FA, FOD and TOD as features for registration.

Figure 2 shows these maps of the averaged deformation distance, masked to the full brain. The figures suggest that the accuracy of FA and FOD registration is relatively flat across the brain, while TOD registration achieves a higher accuracy in dense white matter yet lower in the frontal lobe and the distal gyri. The box plots in the bottom row of Fig. 2 confirm this observation: the median registration accuracy in the full brain mask is $2.52 \mathrm{~mm}$ for FA registration, $2.55 \mathrm{~mm}$ for FOD registration, and $2.45 \mathrm{~mm}$ for TOD registration, but the variability of the TOD registration accuracy is larger. If we define a WM mask by thresholding the track density image (TDI) of each subject at $25 \%$ of its maximum, the accuracy of FA and FOD registration remains approximately unchanged, while the median accuracy of TOD registration drops to $1.51 \mathrm{~mm}$.

\section{Experiment 2: Multiple Subjects}

We applied the presented registration method to the track sets of the 15 different subjects, using the same multi-scale setup and the same parameters as in Experiment 1. The outcome is the mean TOD atlas shown in Fig. 3, as well as 15 deformation fields that map the subjects onto atlas space. Figure 4 shows a subsample of the original, probabilistic track sets of all subjects, and the same tracks warped to atlas space. The 

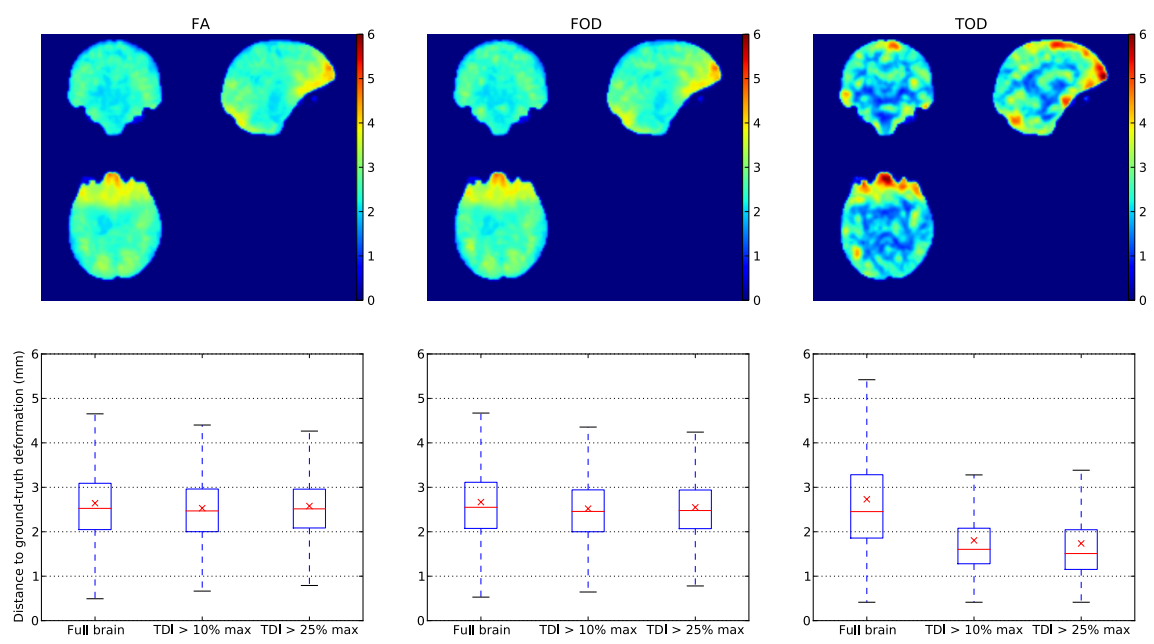

Fig. 2 Results of Experiment 1. Euclidean distance between inter-subject deformation fields and their ground-truths, for FA (left), FOD (middle) and TOD (right) based registration. The top row shows the deformation errors mapped to a common atlas space. The bottom row shows box plots of the registration error in all voxels in a brain mask, and voxels where the track density is larger than $10 \%$ and $25 \%$ of the maximum.

results demonstrate that the dense white matter structures are successfully aligned and clearly reflected in the mean TOD atlas. Remaining alignment errors are located in the caudal end of the corticospinal tract, at the interface of WM and cerebrospinal fluid (CSF) in the ventricles, and in the cortical gyri.

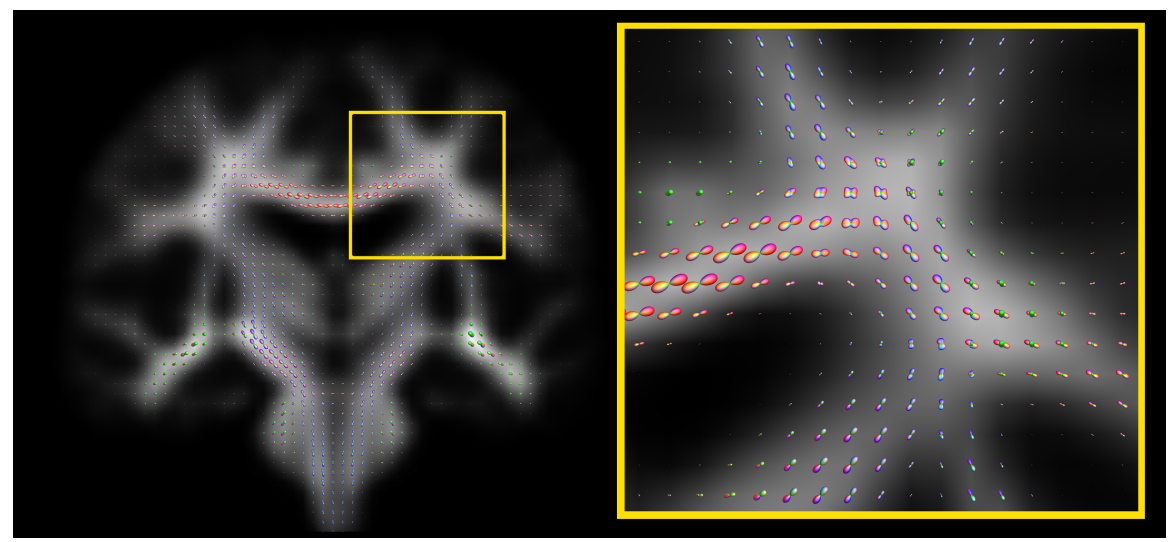

Fig. 3 Results of Experiment 2. Coronal slice of the mean TOD atlas and a close-up of the crossing of the corpus callosum and the corticospinal tract. The 0th order coefficient of the TOD (the TDI map) is overlaid with spherical plots of the TOD in all voxels. 


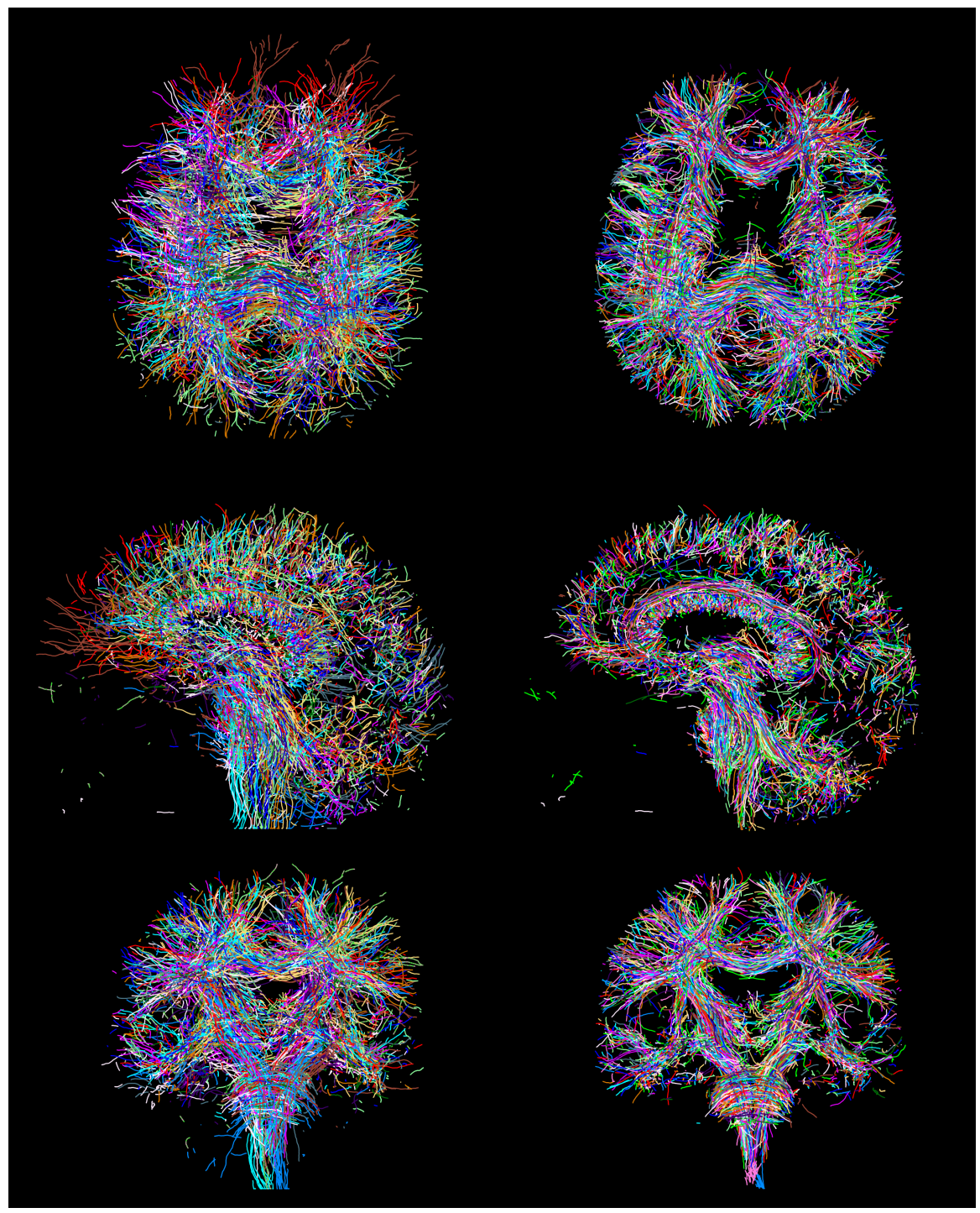

Fig. 4 Results of Experiment 2. Axial, sagittal and coronal slabs (thickness $7.5 \mathrm{~mm}$ ) of the subsampled tractograms (1000 tracks) of all subjects before (left) and after (right) registration. Each subject is represented in a unique colour. 
We repeated this experiment using FA and FOD based registration, like we did in Experiment 1. Next, we use the resulting deformation fields to transform the original tracks of all subjects to the space of each atlas, and recompute the TOD of the transformed tracks at order $\ell_{\max }=8$. Note that this exceeds the order used for registration, and hence contains more detailed angular information. We then assess the quality of the registration by mapping the angular correlation coefficient of the TOD of all subject pairs. The angular correlation coefficient $r_{A}$ between $2 \mathrm{SH}$ functions $U(\boldsymbol{\theta}, \boldsymbol{\phi})=\sum_{i=0}^{n} u_{i} Y_{i}(\boldsymbol{\theta}, \boldsymbol{\phi})$ and $V(\boldsymbol{\theta}, \boldsymbol{\phi})=\sum_{i=0}^{n} v_{i} Y_{i}(\boldsymbol{\theta}, \boldsymbol{\phi})$ is defined as

$$
r_{A}=\frac{\sum_{i=1}^{n} u_{i} \cdot v_{i}}{\sqrt{\sum_{i=1}^{n} u_{i}^{2}} \cdot \sqrt{\sum_{i=1}^{n} v_{i}^{2}}},
$$

and scales between -1 and 1 [1]. The results are displayed in Fig. 5, and show that in deep white matter, i.e., in high track density masks, the angular correlation of the TOD atlas is significantly higher than for the FA and FOD atlases.
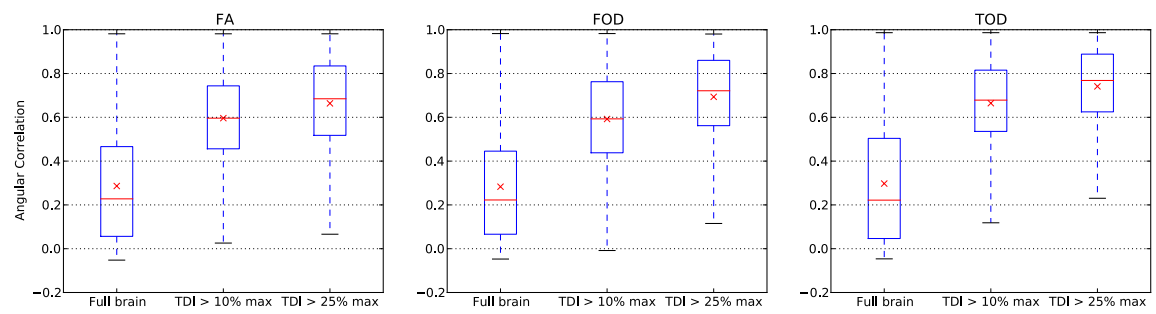

Fig. 5 Results of Experiment 2. Box plots of the angular correlation coefficient between the TOD ( $\mathrm{SH}$ order 8) of the transformed tracks, for FA (left), FOD (middle) and TOD (right) based registration. Box plots are shown for all voxels in a brain mask, and for voxels where the track density is larger than $10 \%$ and $25 \%$ of the maximum.

\section{Discussion}

The TOD offers a new solution to the track set registration problem, akin to the use of Gaussian mixture models in point cloud registration. By modelling the (discrete) tractogram as a (continuous) probability distribution, we can successfully register track sets without relying on correspondences or prior segmentation. This is especially important for the very large (on the order of millions), probabilistic tractography datasets that are increasingly popular.

As opposed to existing track set registration methods, the TOD neglects the longrange connectivity information contained in the track set. The TOD, like the FOD, can not discriminate crossing, kissing and fanning configurations. For the purpose of registration, however, this enhances the robustness against spurious tracks. It is 
precisely because ambiguous FOD configurations often result in false positive tracks, that we wish to exclude long-range information from the matching criterion.

The representational power of the TOD depends on the maximal harmonic order $\ell_{\max }$ of the SH basis, i.e., higher orders allow to model more complex track configurations. For the purpose of registration, however, this high-order information may not contribute much additional information, especially at the expense of the additional computation time that higher orders impose on the registration and reorientation. We therefore selected $\ell_{\max }=4$ as a compromise between both. Fig. 3 shows that 4 th order TODs, while relatively smooth, are sufficient to capture the main directions of crossing bundles.

The experiments show that the presented method can successfully align large probabilistic track sets of 15 subjects. The resulting atlas (Fig. 3) models the expected local track configuration. The mean registration accuracy on data with known ground truth is on the order of the voxel size and improves in regions of high track density, i.e., where the TOD contains the most information. We have compared these results to more conventional image registration methods based on the FA and the FOD, as registration of the underlying DWI data is currently the only alternative to non-rigid alignment of probabilistic track sets. Figure 2 shows that the registration accuracy in the FA and FOD atlas is homogeneous over the whole brain and of the same order as the accuracy of TOD registration. However, the misalignment errors of the TOD registration are localized to regions where the tractogram is sparse (e.g., in the frontal lobe, where magnetic field homogeneities are known to affect the reconstruction). With a track density threshold as low as $10 \%$ of the maximum, the TOD registration significantly outperforms image registration.

With respect to DWI registration in general, the improved quality of the registration in white matter is promising for group analysis of WM-targeted pathologies, although we must stress that the robustness of our method against pathological data has not yet been evaluated. In addition, the sensitivity of TOD registration to the tracking algorithm and its parameters should be investigated prior to such analysis, but goes beyond the scope of this work.

\section{Conclusion and Future Work}

We have presented a novel method for deformable registration of fiber track sets. Rather than comparing individual fibers, we seek to optimise the similarity between the TODs, a spatio-angular probability distribution of the full-brain track configuration, of all subjects. Our method does not require track correspondences or prior bundle segmentation, is robust against interrupted and spurious tracks, and is able to handle crossings and probabilistic tractography data. The resulting population atlas is well suited as prior to track-clustering methods $[9,13,20]$ and for populationbased analysis of the track configuration [8], as it avoids bias towards the underlying DWI data. Indeed, the results indicate that direct registration of the tractograms outperforms conventional image registration methods in dense white matter. 
Several potential improvements remain to be explored. For one, the current similarity measure, i.e., SSD on the TOD coefficients, is sensitive to track density differences and might therefore cause misalignment errors. Entropy- or correlationbased similarity metrics might perform better in that respect. Moreover, similar to the approach taken in Raffelt et al. [15] for FOD registration, the current set-up applies reorientation after each iteration, but ignores it in the calculation of the diffeomorphic update field. Accounting for SH reorientation in the similarity metric is a daunting task and has, to the best of our knowledge, only been done in the LDDMM framework [7]. However, it could lead to faster convergence and more accurate registration, particularly in the distal gyri. Secondly, as the TOD can be represented on an arbitrary voxel grid, future work will investigate if the registration can benefit from a multi-resolution optimisation scheme. Finally, as suggested by Siless et al. [16], T1-weighted images can provide additional contrast in CSF and grey matter, although this requires accurate EPI-distortion correction of the DWI data (e.g., using a reverse-phase encoding acquisition protocol [10]). In fact, as the TOD is represented in the image domain, the proposed method is very well suited to be extended with other modalities.

\section{Acknowledgements}

D. Christiaens is supported by a Ph.D. grant of the Agency for Innovation by Science and Technology (IWT).

\section{References}

1. Anderson, A.W.: Measurement of fiber orientation distributions using high angular resolution diffusion imaging. Magnetic Resonance in Medicine 54(5), 1194-206 (2005)

2. Caan, M.W.A., Van Vliet, L.J., Majoie, C.B.L.M., Van Der Graaff, M.M., Grimbergen, C.A., Vos, F.M.: Nonrigid Point Set Matching of White Matter Tracts for Diffusion Tensor Image Analysis. IEEE Transactions on Biomedical Engineering 58(9), 2431-40 (2011)

3. Calamante, F., Tournier, J.D., Jackson, G.D., Connelly, A.: Track-density imaging (TDI): superresolution white matter imaging using whole-brain track-density mapping. NeuroImage 53(4), 1233-43 (2010)

4. Descoteaux, M., Angelino, E., Fitzgibbons, S., Deriche, R.: Apparent diffusion coefficients from high angular resolution diffusion imaging: estimation and applications. Magnetic Resonance in Medicine 56(2), 395-410 (2006)

5. Dhollander, T., Emsell, L., Van Hecke, W., Maes, F., Sunaert, S., Suetens, P.: Robustifying probabilistic tractography by using track orientation distributions. In: Proc. Intl. Soc. Magn. Res. Med., vol. 21, p. 744 (2013)

6. Dhollander, T., Veraart, J., Van Hecke, W., Maes, F., Sunaert, S., Sijbers, J., Suetens, P.: Feasibility and Advantages of Diffusion Weighted Imaging Atlas Construction in Q-Space. In: G. Fichtinger, A. Martel, T. Peters (eds.) MICCAI 2011, LNCS, vol. 6892, pp. 166-73. Springer, Heidelberg (2011) 
7. Du, J., Goh, A., Qiu, A.: Diffeomorphic Metric Mapping of High Angular Resolution Diffusion Imaging based on Riemannian Structure of Orientation Distribution Functions. IEEE Transactions on Medical Imaging 31(5), 1021-33 (2012)

8. Durrleman, S., Fillard, P., Pennec, X., Trouvé, A., Ayache, N.: Registration, atlas estimation and variability analysis of white matter fiber bundles modeled as currents. NeuroImage 55(3), 1073-90 (2011)

9. Guevara, P., Duclap, D., Poupon, C., Marrakchi-Kacem, L., Fillard, P., Le Bihan, D., Leboyer, M., Houenou, J., Mangin, J.F.: Automatic fiber bundle segmentation in massive tractography datasets using a multi-subject bundle atlas. NeuroImage 61(4), 1083-99 (2012)

10. Holland, D., Kuperman, J.M., Dale, A.M.: Efficient correction of inhomogeneous static magnetic field-induced distortion in Echo Planar Imaging. NeuroImage 50(1), 175-83 (2010)

11. Mayer, A., Zimmerman-Moreno, G., Shadmi, R., Batikoff, A., Greenspan, H.: A supervised framework for the registration and segmentation of white matter fiber tracts. IEEE Transactions on Medical Imaging 30(1), 131-45 (2011)

12. O'Donnell, L.J., Wells, W.M., Golby, A.J., Westin, C.F.: Unbiased groupwise registration of white matter tractography. In: N. Ayache, H. Delingette, P. Golland, K. Mori (eds.) MICCAI 2012, LNCS, vol. 7512, pp. 123-30. Springer, Heidelberg (2012)

13. O'Donnell, L.J., Westin, C.F.: Automatic Tractography Segmentation Using a HighDimensional White Matter Atlas. IEEE Transactions on Medical Imaging 26(11), 1562-75 (2007)

14. Raffelt, D., Tournier, J.D., Crozier, S., Connelly, A., Salvado, O.: Reorientation of fiber orientation distributions using apodized point spread functions. Magnetic Resonance in Medicine 67(3), 844-55 (2012)

15. Raffelt, D., Tournier, J.D., Fripp, J., Crozier, S., Connelly, A., Salvado, O.: Symmetric diffeomorphic registration of fibre orientation distributions. NeuroImage 56(3), 1171-80 (2011)

16. Siless, V., Glaunès, J., Guevara, P., Mangin, J.F., Poupon, C., Le Bihan, D., Thirion, B., Fillard, P.: Joint $\mathrm{T} 1$ and brain fiber log-demons registration using currents to model geometry. In: N. Ayache, H. Delingette, P. Golland, K. Mori (eds.) MICCAI 2012, LNCS, vol. 7511, pp. 57-65. Springer, Heidelberg (2012)

17. Tournier, J.D., Calamante, F., Connelly, A.: Robust determination of the fibre orientation distribution in diffusion MRI: non-negativity constrained super-resolved spherical deconvolution. NeuroImage 35(4), 1459-72 (2007)

18. Tournier, J.D., Calamante, F., Connelly, A.: MRtrix: Diffusion tractography in crossing fiber regions. International Journal of Imaging Systems and Technology 22(1), 53-66 (2012)

19. Vercauteren, T., Pennec, X., Perchant, A., Ayache, N.: Diffeomorphic demons: efficient nonparametric image registration. NeuroImage 45(1), S61-S72 (2009)

20. Wassermann, D., Bloy, L., Kanterakis, E., Verma, R., Deriche, R.: Unsupervised white matter fiber clustering and tract probability map generation: applications of a Gaussian process framework for white matter fibers. NeuroImage 51(1), 228-41 (2010)

21. Ziyan, U., Sabuncu, M.R., O’Donnell, L.J., Westin, C.F.: Nonlinear registration of diffusion MR images based on fiber bundles. In: N. Ayache, S. Ourselin, A. Maeder (eds.) MICCAI 2007, LNCS, vol. 4791, pp. 351-8. Springer, Heidelberg (2007)

22. Zvitia, O., Mayer, A., Shadmi, R., Miron, S., Greenspan, H.K.: Co-registration of White Matter Tractographies by Adaptive-Mean-Shift and Gaussian Mixture Modeling. IEEE Transactions on Medical Imaging 29(1), 132-45 (2010) 